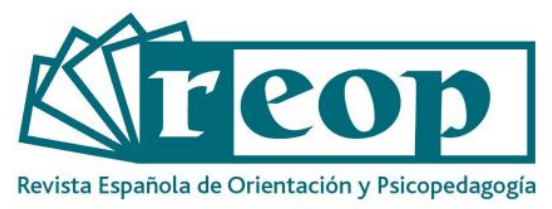

\title{
UNA INVESTIGACIÓN SOBRE LAS TUTORIAS COMPARTIDAS
}

\section{A RESEARCH ON SHARED TUTORIAL ACTION}

\author{
Ángela Saiz Linares ${ }^{1}$ \\ Universidad de Cantabria. Facultad de Educación. Departamento de Educación. Santander, \\ España \\ Noelia Ceballos López \\ Universidad de Cantabria. Facultad de Educación. Departamento de Educación. Santander, \\ España
}

\section{RESUMEN}

Esta investigación tiene por objetivo analizar, desde una perspectiva etnográfica, un caso paradigmático de "escuela orientadora" que sitúa la acción tutorial como pilar sobre el que apuntalar diferentes acciones y propuestas educativas a partir de un proyecto de tutorías compartidas. En este trabajo de investigación examinamos algunas claves que permiten tender puentes entre la definición canónica de acción tutorial y el proyecto educativo de esta escuela. Las técnicas de producción de información han sido: observación participante, grupos de discusión con docentes (tutores, orientadora y equipo directivo) y con alumnado de Educación Primaria (desde $1^{\circ}$ a $6^{\circ}$ ), entrevistas semiestructuradas con tutores y análisis documental (PEC, PAT y PIIE). A partir de un análisis de contenido tratamos de dar vida a los presupuestos que la literatura ha confirmado como irrenunciables: institucionalizar oportunidades de convivencia inter nivelares y con la comunidad; trabajar consistentemente las dimensiones personales, educativas, profesionales y socio-cívicas; crear tiempos y espacios destinados al intercambio reflexivo entre profesionales educativos. Concluimos confirmando que la interseccionalidad de las acciones analizadas permite abordar sistemáticamente las dimensiones que vertebran los proyectos de acción tutorial.

\footnotetext{
${ }^{1}$ Correspondencia: Ángela Saiz-Linares. Correo-e: saizla@unican.es
} 
Palabras clave: Acción tutorial, escuela orientadora, tutorías compartidas, educación inclusiva, etnografía.

\begin{abstract}
This research aims to analyze, from an ethnographic perspective, a paradigmatic case of a "counselling school" that places Tutorial Action as a pillar on which different educational actions and proposals are based. In this research, we examine some key issues that allow us to build bridges between the canonical definition of Tutorial Action and the educational project of this school. The information production techniques were participant observation, focus groups with teachers (tutors, counsellor, and management team), and primary school students (from 1st to 6th grade), semi-structured interviews and document analysis. From a content analysis we try to give life to the premises that literature has confirmed as indispensable: institutionalize opportunities for coexistence between different levels and with the community; consistently work on personal, educational, professional, and socio-civic dimensions; create times and spaces for reflective exchange between professionals. We conclude by confirming that the intersectionality of the actions analyzed allows us to work consistently on the dimensions that structure the Tutorial Action projects.
\end{abstract}

Key Words: Tutorial action, educational counselling, shared tutoring, inclusive education, ethnography.

\title{
Cómo citar este artículo:
}

Saiz-Linares, Á. y Ceballos, N. (2021). Una investigación sobre tutorías compartidas. Revista Española de Orientación y Psicopedagogía, $32(1), \quad 48$. https://doi.org/10.5944/reop.vol.32.num.1.2021.30739

\section{Introducción}

En este artículo analizamos un ejemplo paradigmático de "escuela orientadora" (Mollá y Longás, 2007) que sitúa a la acción tutorial (AT) y la atención a la diversidad como pilares sobre los que apuntalar las propuestas educativas del centro. Tomaremos como referencia la definición de AT acuñada por Álvarez (2017), cuya enunciación recoge la complejidad de dicho proceso y los diferentes componentes reconocidos como fundamentales (Álvarez, 2006; Álvarez y Bisquerra, 2012; Gallego y Riart, 2006; Lázaro y Asensi, 1987; Sanz, 2010). Pretendemos poner en diálogo el contenido de este enunciado con los resultados de la investigación en esta "escuela orientadora", mostrando prácticas curriculares y organizativas que ilustren las diferentes aristas conceptuales de las que consta esta definición:

Acción formativa de orientación, ayuda y mediación que el profesor-tutor y el resto del equipo docente realiza con su alumnado a nivel individual y grupal en el ámbito personal, escolar, profesional y social, al mismo tiempo que ejercen su función docente. Esta acción formativa ha de dirigirse también y ha de contemplar 
la colaboración del resto de agentes educativos, particularmente de las familias (Álvarez, 2017, p.30).

Nos servimos de una investigación realizada en el CEIP Manuel Llano (Santander, España) que, desde hace unos años, desarrolla un proyecto de acción tutorial internivelar (tutorías compartidas) que ha penetrado hondamente en la cultura escolar y sirve de marco para otras acciones educativas. El Proyecto Educativo del centro se anuda con el compromiso de atender a la diversidad para buscar una enseñanza de calidad para todos los alumnos. Al abrigo de este compromiso, desarrolla un proyecto en el marco de la AT que implica a todo el centro. Para este proyecto se genera un espacio de trabajo inter-nivelar (grupos con alumnos de diferentes cursos acompañados por un docente distinto a su tutor), grupos que quincenalmente desarrollan actividades en torno a diferentes temáticas (igualdad de género, interculturalidad, los objetivos de desarrollo sostenible (ODS)...), que persiguen generar situaciones de encuentro, cooperación y reconocimiento.

\section{Marco teórico}

El paradigma de escuela orientadora (Mollá y Longás, 2007) adquiere relevancia en una sociedad actualmente marcada por la agenda neoliberal, donde el modelo de escuela se orienta hacia objetivos instruccionales y competitivos, delineados al amparo de las evaluaciones externas. En este contexto, la escuela se ve interpelada a repensar su sentido y organización para responder a nuevas situaciones, aprendizajes y modos de relacionarse (Expósito, 2014) con el fin de: construir escuelas que aseguran la presencia, participación y éxito de todos los alumnos sin excepción (Ainscow et al., 2006); comprometerse con la formación de ciudadanos que contribuyan a una sociedad más justa y democrática; y utilizar la vida cotidiana como proyecto educativo preponderante (las relaciones, la participación y toma de decisiones). En definitiva, la escuela orientadora se define como un elemento clave en la reconfiguración de las escuelas.

La Orientación Educativa no tiene un significado unívoco (Monescillo, 2005). Es recientemente cuando emerge como un proceso de ayuda continuo y sistemático, dirigido a todas las personas, en todos sus aspectos, poniendo especial énfasis en la prevención y el desarrollo, que se realiza a lo largo de toda la vida, con la implicación de los diferentes agentes educativos y sociales (Álvarez y Bisquerra, 2012). Esta se ha materializado en diferentes niveles de acción, siendo la AT el primer nivel de desarrollo que sitúa al aula y al centro como contextos de actuación. Es en este nivel donde se enmarca nuestro trabajo.

La AT es un proceso inherente a la acción educativa y, por ende, a la acción docente. En consecuencia, es responsabilidad de todo el profesorado, bajo la coordinación del tutor (Álvarez, 2006), llevar a cabo procesos de ayuda, orientación y seguimiento del proceso académico y personal del alumnado (Blanchard, 2010).

Por otro lado, es un proceso continuo y sistemático cuya finalidad debe orientarse al pleno desarrollo del alumnado en el plano individual y colectivo. En el individual, la tutoría se entiende como un proceso de seguimiento, apoyo y personalización del desarrollo integral, que brota en la interacción entre el tutor y el alumno y donde las actuaciones deben orientarse a: conocer las particularidades individuales para ofrecer a cada alumno diferentes planteamientos metodológicos y curriculares que tengan en cuenta sus aptitudes, intereses y motivaciones; efectuar un seguimiento de su desarrollo e intensificar los recursos existentes; y favorecer un trabajo colaborativo entre los diferentes agentes de intervención. Esta AT individual debe estar al servicio 
de todo el alumnado, no únicamente de aquellos que muestran más dificultades académicas (Besalú, 2010). De otro lado, es imperativo ofrecer actuaciones que se desarrollan en un nivel grupal. Algunas actuaciones en esta línea tienen que ver con: conocer la dinámica interna del grupo, promover actividades que fomenten la convivencia, la integración y la participación de los alumnos en la vida del centro, prevenir y gestionar los conflictos, etc.

Abundantes autores han tratado de definir y concretar las dimensiones de la AT (Álvarez, 2006; Álvarez y Bisquerra, 2012; Arnáiz, 2001; Ceballos, 2017; Gallego y Riart, 2006; Galve, 2002; Torrego et al., 2014; Santana, 2012), donde todos comparten la necesidad de abordar los ámbitos personal, educativo, profesional y social (Tabla 1):

\section{Tabla 1.}

Las dimensiones de la Acción Tutorial.

\begin{tabular}{|c|c|}
\hline $\begin{array}{l}\text { Aprender a } \\
\text { comprender el } \\
\text { mundo }\end{array}$ & $\begin{array}{l}\text { - Conocer y comprender el mundo como una construcción que reproduce } \\
\text { la discriminación existente. } \\
\text { - Aportar una mirada crítica a la cultura existente. } \\
\text { - Problematizar el conocimiento y abordar problemáticas sociales. } \\
\text { - Desarrollar preguntas críticas acerca del mundo. Abordar la búsqueda } \\
\text { de respuestas desde procesos de investigación y análisis profundos. }\end{array}$ \\
\hline Aprender a ser & $\begin{array}{l}\text { - Proceso de construcción de su propia identidad: } \\
\text { - Vertiente personal. Conocerse a sí mismo y construir una imagen } \\
\text { positiva y ajustada: personal, étnica, académica, sexual, de género, } \\
\text { cultural, etc. } \\
\text { - Vertiente social: libertad, justicia y respeto mutuo. }\end{array}$ \\
\hline $\begin{array}{l}\text { Aprender a } \\
\text { convivir }\end{array}$ & $\begin{array}{l}\text { - Desarrollar principios de convivencia como la justicia, la participación y } \\
\text { el respeto. } \\
\text { - Conocer al otro y su historia favoreciendo la comprensión mutua y el } \\
\text { respeto. } \\
\text { - Aprender a trabajar juntos, compartir proyectos y objetivos comunes. } \\
\text { - Modelo de convivencia escolar integrado. }\end{array}$ \\
\hline $\begin{array}{l}\text { Aprender a } \\
\text { actuar }\end{array}$ & $\begin{array}{l}\text { - Más allá de una cualificación profesional. } \\
-\quad \text { Conocer sus fortalezas y competencias personales. } \\
\text { - Análisis de los sistemas de producción y las relaciones laborales. } \\
\text { - Responder a situaciones sociales complejas. } \\
\text { compartida. }\end{array}$ \\
\hline $\begin{array}{l}\text { Aprender a } \\
\text { tomar decisiones }\end{array}$ & $\begin{array}{l}\text { - Participar e implicarse en la toma de decisiones de los aspectos } \\
\text { relevantes de su vida. } \\
\text { - La escuela como espacio donde vivir la democracia: diálogo y toma de } \\
\text { decisiones compartidas. } \\
\text { - Prevalencia de la búsqueda del bien común y la valentía cívica. }\end{array}$ \\
\hline
\end{tabular}

Fuente: elaboración propia partiendo de la propuesta de Ceballos (2017).

Además, cabe señalar que la AT es una labor pedagógica en colaboración con otros miembros de la comunidad educativa (Álvarez y Bisquerra, 2012), donde destaca el papel de las familias: "entre las familias y el centro debe haber una relación fluida, procurando que éstas se impliquen en el proceso de formación del alumno" (Sanz, 2010, p. 10). Estudios previos muestran cómo, a pesar de ser una acción deseada y destacada por su potencial en el desarrollo y aprendizaje del alumnado, la implicación de las familias en el proceso educativo se circunscribe a acciones puntuales y de intercambio de información (Ceballos y Saiz, 2019). Asimismo, si atendemos a su participación en la organización y funcionamiento del centro encontramos que esta se circunda a su presencia en los órganos formales, donde su participación es más simbólica 
que real, o al ámbito extracurricular (salidas o fiestas) (Ceballos y Saiz, 2019; Méndez et al., 2010). Es importante dejar atrás estos planteamientos restrictivos y engendrar relaciones sustentadas en un paradigma colaborativo donde compartir metas y tomar decisiones juntos (García-Albaladejo y Sánchez-Liarte, 2006; Llevot y Bernad, 2015).

Finalmente, en un proyecto dirigido al desarrollo integral del alumnado, cobra vital importancia la colaboración con la comunidad local (Cieza, 2010; Simón, 2011). Parrilla et al. (2013) señalan dos perspectivas a este respecto: la escuela al servicio de la comunidad socioeducativa (Cummings y Dyson, 2007) y la escuela como parte integrante e integral del desarrollo comunitario (Bottrell y Goodwin, 2011). Esta última requiere la apertura de la escuela a la comunidad y realidad social y la implicación de la comunidad local en las acciones educativas de la escuela (Longás et al., 2008). Debemos abandonar la inercia existente que señala a la escuela como respuesta de todas las demandas sociales y caminar hacia la colaboración tejiendo corresponsabilidades socioeducativas (Parrilla et al., 2013).

\section{Método}

Desde el punto de vista metodológico, esta investigación se incardina en la tradición cualitativa con una perspectiva etnográfica (Beach et al., 2018; Restrepo, 2016). La etnografía educativa posibilita realizar un estudio y descripción de dicha cultura analizando la institución escolar y las prácticas que acaecen dentro de ella (Serra, 2004; Álvarez, 2011; Cotán, 2020). De esta suerte, este enfoque nos permite describir, comprender e interpretar en profundidad y desde "dentro" la realidad del centro educativo que analizamos a partir de las relaciones humanas y su contexto (Restrepo, 2016). Concretamente el propósito de nuestra investigación es comprender, desde una perspectiva de interpretación crítica, las actuaciones de AT del centro en el marco de las tutorías compartidas y las condiciones que posibilitan convertir estos ámbitos en el corazón del resto de actuaciones escolares poniendo rumbo hacia una escuela orientadora.

Nuestra relación con dicho centro se inició en 2010, cuando nuestro equipo de investigación emprendió con la dirección del centro y algunos docentes un proyecto para estimular procesos de cambio vinculados a la participación del alumnado ${ }^{2}$ guiados por los principios de inclusión educativa. Merced a esta investigación, la escuela inició un proceso de reflexión y replanteamiento sobre muchas acciones y dinámicas desde las claves inclusivas. Esta colaboración se prolongó durante 6 años, tras lo cual el equipo de investigación fue paulatinamente replegándose y transfiriendo la responsabilidad de las iniciativas de participación a los agentes del centro. Ocho años después de su inicio dos investigadoras del equipo retornamos al centro con objeto de analizar uno de los proyectos de participación del alumnado que germinó en esos años de colaboración y que ahora constituye una de las señas de identidad del centro: las tutorías compartidas.

Esta relación estrecha con el equipo directivo facilitó la negociación y el acceso al campo, que fue un proceso amable y realizado con bastante presteza. Tras explicar el propósito de nuestra investigación y delinear cómo sería la presencia de las investigadoras, procedimos a negociar los momentos de observación que sucederían y otros instrumentos de producción de información que utilizaríamos (grupos de discusión y entrevistas).

\footnotetext{
${ }^{2}$ Susinos, T. (Dir.). Análisis de los procesos de inclusión/exclusión educativa en la educación obligatoria. Desarrollo de proyectos locales de cambio y mejora escolar. MICINN, Ref. EDU2008-06511-C02 02/EDUC.
} 
Realizamos la fase de trabajo de campo en el curso académico 2017-2018, durante el cual realizamos visitas sistemáticas y abundantes al centro educativo para evitar una "etnografía relámpago" (Rist,1980). En otras palabras, las investigadoras desarrollamos una participación prolongada en el contexto para tratar de comprender y describir lo que sucede desde el punto de vista de sus miembros (Álvarez, 2011). Por tanto, si bien como observadoras externas tratamos de implicarnos lo menos posible en las experiencias educativas que observamos, establecemos una vinculación fluida con las personas que forman parte del centro con objeto de recoger información sobre las acciones y significados en su ambiente natural e interpretar dicha información en contexto (Serra, 2004).

\section{Muestra}

Nuestra muestra de estudio refiere, por tanto, al conjunto profesionales, alumnos y otros agentes que participan habitualmente en las tutorías compartidas del CEIP Manuel Llano, en el que conviven 332 alumnos y 33 profesores. Se trata de un centro de titularidad pública, situado en una zona periférica de Santander en la que confluyen varios barrios de características diversas y que da lugar a que el alumnado que acoge el centro sea heterogéneo en cuanto a su origen social, cultural y económico. Su proyecto educativo (PEC) presenta un compromiso explícito con la atención a la diversidad desde una perspectiva inclusiva. Esto los lleva a reflexionar constantemente sobre la organización escolar, el currículum, las relaciones y/o los procesos de aprendizaje, buscando constantemente aumentar el nivel de participación de todos sus miembros en aras de convertir el centro en una escuela para todos.

La iniciativa de las tutorías compartidas sigue esta estela inclusiva. Este proyecto, encuadrado en el marco de la acción tutorial y que originalmente advino como un proyecto de nivel desarrollado en $5^{\circ}$ de Primaria, se orienta a mejorar la convivencia e implica desde el curso 20142015 a todos los docentes y alumnado de educación primaria.

Para ello se genera un espacio de trabajo inter-nivelar (en total, 12 grupos con alumnos de diferentes cursos) que ocurre quincenalmente para realizar actividades sobre diferentes temáticas (escogidas por su relevancia social y por su trascendencia para la atención a la diversidad: igualdad de género, interculturalidad, los ODS...) que persiguen generar situaciones que contribuyan a la construcción de una identidad positiva, la cooperación, el respeto, el desarrollo del sentido de pertenencia y, en resumidas cuentas, apoyar, atender y celebrar la diversidad.

\section{Instrumentos}

Las técnicas de producción de datos son las convenientes dentro de este paradigma:

- 8 observaciones participantes (acudimos al centro para observar cada una de las tutorías compartidas), entendidas como procesos intencionales y sistemáticos, que plasmamos en notas de campo y diarios (Rozsahegyi, 2019). Esta periodicidad permitió sostener un contacto constante con los sujetos observados, así como recoger información en su medio natural (Velasco y Díaz de Rada, 2006). En estas observaciones participamos ambas investigadoras, comprendiendo más contextos de observación, lo que nos ha permitido observar, al menos una vez, a todos los grupos de tutorías compartidas conformados. 
- 4 grupos de discusión (GD), de carácter semiestructurado, de 6 participantes cada uno se llevaron a cabo al finalizar el proyecto con intención de evaluar la iniciativa (Kamberelis et al., 2011).

- Dos GD estuvieron conformados por profesorado: en uno participaron 5 tutores y la orientadora del centro y, en el otro, 3 tutores, la jefa de estudios, la directora y uno de los integrantes del equipo impulsor de tutorías compartidas. Se organizaron en torno a los siguientes bloques temáticos: objetivos y decisiones iniciales, aspectos organizativos, tutorías compartidas de 2017/2018 (temática, actividades, participación de los diferentes agentes), aprendizajes desarrollados.

- Los otros dos GD estuvieron constituidos por alumnado (cada grupo tenía representación de alumnos desde $1^{\circ}$ de Primaria hasta $6^{\circ}$ ) y los bloques temáticos de que constaron fueron: aspectos organizativos, actividades realizadas y cambios futuros.

La duración aproximada de los GD con profesionales del centro fue de 90 minutos y con el alumnado de unos 45. Todos se llevaron a cabo en diferentes espacios del centro escolar (en aulas y en la biblioteca). Fueron grabados y posteriormente transcritos.

- 1 entrevista semiestructurada a una informante clave, concretamente a la otra integrante del equipo impulsor del proyecto (Kvale, 2011) y profesora más veterana del centro. La entrevista, comprendida como espacio de comunicación intencionada, con una estructura y un propósito previamente determinado, nos permitió acceder a datos complementarios a los GD y matizar algunas informaciones (profundizamos sobre condiciones de la organización escolar que han posibilitado institucionalizar el proyecto de las Tutorías Compartidas). Al igual que los GD, esta fue grabada en audio y transcrita para su análisis y su duración fue de una hora y media aproximadamente.

- Análisis de documentos institucionales del centro (Proyecto Educativo de Centro y Plan de Acción Tutorial y PIIE ${ }^{3}$ ) (O'Connor, 2019). Permitió conocer información relevante sobre el proyecto y contrastar la información obtenida con el resto de las estrategias.

\section{Análisis}

Hemos obtenido una extensa diversidad de información, aportada por las diferentes miradas para comprender la complejidad de la realidad estudiada. La etnografía educativa no debe, no obstante, circunscribirse exclusivamente a la dimensión descriptiva de la cultura y las dinámicas escolares, sino que ha de coadyuvar también a realizar interpretaciones teóricas y prácticas conducentes a una intervención pedagógica mejor (Woods, 1987). Para realizar esta interpretación de la información obtenida realizamos un proceso de codificación temática (Flick, 2018). Este se confeccionó a partir de procesos inductivos, partiendo de un esquema de conceptos definidos, guiándonos por los datos, y deductivos, que emergen de la revisión bibliográfica. De esta suerte, las categorías de nuestro análisis se corresponden con los cuatro ejes conceptuales de que consta la definición de acción tutorial enunciada por Álvarez (2017, p. 30): "Acción formativa de orientación, ayuda y mediación...":

- Que el profesor-tutor y el resto del equipo docente (responsabilidad de la AT: categoría 1).

\footnotetext{
${ }^{3}$ Los PIIE son los Proyectos Integrados de Innovación Educativa convocados por la Consejería de Educación de Cantabria.
} 
- Realiza con su alumnado a nivel individual y grupal (destinatarios de la AT: categoría 2).

- En el ámbito personal, escolar, profesional y social (dimensiones de la AT: categoría 3).

- Que ha de dirigirse también y ha de contemplar la colaboración del resto de agentes educativos, particularmente de las familias (relaciones con otros agentes en el marco de la AT: categoría 4).

\section{Resultados y discusión}

Organizamos los resultados a través de las características de la acción tutorial presentes en la definición propuesta por Álvarez (2017), con el fin de visibilizar cómo las prácticas curriculares y organizativas que se despliegan en la escuela estudiada, en el marco de las tutorías compartidas, responden a dicha definición.

\section{Acción tutorial... "que el profesor-tutor y el resto del equipo docente realiza"}

La literatura pedagógica y la legislación vigente consideran la AT como una labor que incumbe a todo el profesorado y que resulta inherente a la acción docente del tutor, contando con la cooperación del equipo docente y el respaldo de la institución escolar.

En la escuela analizada la organización se pone al servicio de esta premisa, de modo que todos los profesores acompañan al estudiante en su desarrollo con acciones de diversa naturaleza. Por un lado, encontramos una AT continúa ejercida por el tutor y el equipo docente de cada grupo, estableciéndose una hora semanal específica, no contemplada en la normativa, destinada a la tutoría con objeto de sistematizar el abordaje de actividades relacionadas con la gestión del grupo-clase, con el desarrollo personal y social, etc.

"En el centro consideramos la acción tutorial una de las labores más complejas y prioritarias de la función docente. Supone, en sí misma un pilar fundamental sobre el que apoyar el resto de las labores docentes y educativas. Es por ello por lo que apostamos por incluir en nuestro horario semanal una sesión de "tutoría". Entendemos que la acción tutorial se establece de manera continua a lo largo de todo el horario lectivo, en cambio, vemos el establecimiento de esta sesión como una estrategia para crear un espacio que nos ayude a sistematizar el abordaje de actividades concretas relacionadas con la gestión del grupo-clase, con el desarrollo personal y social y con temáticas que atiendan las necesidades e intereses de nuestro alumnado." (PAT).

Esta AT ordinaria se complementa con los encuentros y actividades destinados a las tutorías compartidas, donde se fracturan estos grupos de referencia graduados por edad para construir otros de carácter internivelar, que cuentan también con una figura docente distinta a su tutor. Esta reordenación de grupos y tutores engendra efectos a nivel relacional: se intensifican las oportunidades de encuentro y se estrechan las relaciones y vínculos entre docentes y niños de cursos diferentes, posibilitando que todo el profesorado pueda ofrecer cuidado, orientación y ayuda a todos los niños, formen o no parte de su grupo-aula. 
Dentro de tu grupo de tutorías compartidas tienes niños desde primero hasta sexto. Entonces hay profesores que nunca han convivido con ese niño de primero que en ese momento sí que lo tienen. Eso también te ayuda a la organización, a las actividades en el patio... (GD_profesorado2).

Para esta tarea en las tutorías compartidas resulta fundamental la cooperación de docentes que comparten el horizonte de atender a la diversidad desde un marco inclusivo. Las observaciones nos llevan a confirmar la existencia de una cultura común donde estos compromisos se comparten. Esta cultura de la participación es fruto de un proceso de reflexión y acción extenso, liderado por el equipo directivo, donde se han creado estructuras facilitadoras de diálogo y de reflexión compartida, que han posibilitado la implicación de todos los docentes en la tarea de pensar cómo ofrecer una escuela para que todos los alumnos tengan éxito.

Yo creo que la clave es generar espacios en el centro (a nivel lectivo) y espacios de participación entre el profesorado. Si no generas esos espacios te come el día a día. Necesitas un espacio para pensar y diseñar esas actividades. Nuestro modelo de tutorías sin un espacio para compartir y para preparar, es imposible. (GD_profesorado1).

Una de las estructuras creadas para este propósito es los "equipos impulsores", conformados por 2 ó 3 docentes, que buscan la participación de todo el profesorado en la tarea de repensar las prácticas educativas hacia el faro de la atención a la diversidad.

"La autonomía que tenemos nos permite [...] generar estructuras, espacios y tiempos para coordinar y reflexionar sobre la práctica docente. Destacamos las coordinaciones horizontales y verticales que nos han permitido adoptar acuerdos tanto a nivel de ciclo/nivel como de centro. Así mismo, destacamos la figura de los equipos impulsores (internivelares de profesores) que permiten compartir responsabilidades y organizar el trabajo colaborativo dinamizando e integrando las diferentes competencias clave. (PEC).

Actualmente funcionan cinco equipos impulsores: Competencia lingüística, competencia matemática, tutorías compartidas y convivencia, bilingüe y de salud. Como vemos, uno de ellos, liderado por dos docentes, se dedica específicamente a planear actuaciones en el marco de las tutorías compartidas. Para que esto sea posible, cuentan dentro de su horario lectivo con tiempos que les permiten establecer espacios para encontrarse, coordinarse, reflexionar e investigar conjuntamente.

Para cada uno de los equipos tenemos generados unos espacios y tiempos para que se reúnan. En el caso de las Tutorías Compartidas, son un equipo internivelar y tienen unos momentos en los que se reúnen para coordinar, organizar esto... (GD_profesorado1).

De otro lado, los equipos impulsores se erigen en una estrategia valiosa para integrar al profesorado nuevo. El claustro en los últimos años ha sido fuertemente variable, debido a numerosas jubilaciones y al notable porcentaje de profesorado interino. Estos nuevos docentes reciben siempre acompañamiento de los equipos impulsores (en las tutorías compartidas los nuevos docentes han sido emparejados con otros más veteranos) y son, además, invitados expresamente a contribuir con sus experiencias y conocimientos al proyecto educativo del centro.

Llego al aula donde está el segundo grupo. Está una maestra y la orientadora. [...] Al final de la sesión, pregunto por qué son dos. La orientadora me explica que la otra docente es nueva en el centro y que, a modo de acompañamiento, siempre emparejan a docentes recién llegados con aquellos que conocen el proyecto. (Observación4). 
En síntesis, la vocación participativa ha servido de estímulo para generar estructuras de trabajo horizontales destinadas a generar procesos de reflexión e innovación en distintos ámbitos, como es el caso de la acción tutorial, lo que resulta en el involucramiento activo de todo el profesorado en la labor orientadora.

\section{Acción tutorial... "con su alumnado a nivel individual y grupal"}

En el CEIP Manuel Llano, "la orientación pretende contribuir al desarrollo integral de todos los alumnos" (PEC Manuel Llano). Para ello, el centro escolar pone en marcha actuaciones individuales que facilitan la personalización del proceso educativo, con objeto de mejorar la atención a la diversidad del alumnado para que todos los niños/as, sin excepción, tengan éxito en sus aprendizajes. Partiendo de la base de que "cada alumno es diferente en cuanto a ritmos, estilos cognitivos, intereses, etc., en el centro se aboga por abordar el currículo desde metodologías activas y participativas que permitan responder a todo el estudiantado" (PEC Manuel Llano). En este sentido, la AT se establece de manera continua a lo largo de todo el horario lectivo.

Durante las tutorías compartidas se reformulan los grupos-aula de referencia para religar a alumnado de diferentes niveles, componiendo nuevos grupos de 15 alumnos en los que conviven niños/as de $1^{\circ}$ hasta $6^{\circ}$ de Primaria. Estos grupos que se crean no son azarosos. El equipo impulsor de las tutorías compartidas imparte clases en todos los cursos, de manera que tienen conocimiento personal de todos los niños/as. Con ello, estos maestros destinan un tiempo grande a reflexionar todos los años sobre cómo puede funcionar cada alumno/a en los diferentes grupos, siempre bajo el criterio de favorecer la convivencia:

Encontramos un grupo heterogéneo formado por niñas y niños de los diferentes cursos [...]. Observo que hay un alumno que requiere la atención constante de la docente. Converso con la tutora sobre ello y me explica que M. está en ese grupo por la relación establecida con ella y su dinámica de trabajo. (Diario de investigación).

Esta experiencia internivelar no surge de manera inmediata. Como la mayoría de las escuelas urbanas, esta viene de una tradición graduada fuertemente asentada en la cultura del centro, por lo que su propensión inaugural fue la de entremezclar a alumnos de diferentes niveles, pero sin una distancia excesiva con respecto a la edad. Sin embargo, el tiempo y la reflexión compartida sobre la experiencia han permitido acrisolar la iniciativa y arriesgarse hacia otras formas de agrupamiento más diversas.

Al principio [los primeros años de tutorías compartidas] mezclábamos de otra manera diferente. En vez de todos los cursos, poníamos a $2^{\circ}$ con $4^{\circ}$ pero vimos que era más enriquecedor tener todas las edades. (GD_profesorado2)

Estos grupos de naturaleza heterogénea se convierten en una oportunidad para construir nuevos vínculos de apoyo entre alumnado de diferentes edades y mejorar, consecuentemente, la convivencia y el sentido de pertenencia:

Toca el timbre y se comienza a escuchar bullicio. Los alumnos/as salen de sus aulas y se desplazan por las diferentes plantas del edificio hacia el aula que les corresponde. Unas pocas maestras se encuentran en los pasillos atentas a cómo trascurre. Cuando ven a algún niño desorientado le ayudan, pero prácticamente no intervienen, son los alumnos de cursos superiores quienes acompañan a los de cursos inferiores. (Observación 6).

Los lazos de cooperación que se crean en las tutorías compartidas se extienden a otros momentos y espacios conformando un modo de vida comunitario donde todos se conocen, se 
apoyan y nadie se encuentra solo. La estrategia de interacción internivelar forma parte de un entramado de acciones destinadas a generar un clima relacional donde prevalece la reciprocidad y que pretenden transformar al centro en un entorno seguro y acogedor que garantiza la participación y el aprendizaje de todos los alumnos.

La creación de un ambiente de relaciones, una manera de hacer que no solo se circunscribe a las tutorías compartidas, sino que, a lo largo del año, en otro montón de actividades se lleva a cabo: "apadrina un lector", "dime algo bueno de ti”. (GD_profesorado1).

A mí me parece muy bien porque estamos todos juntos, los niños más mayores con los más pequeños. Y aunque haya cosas más difíciles los mayores nos ayudan a hacerlo. (GD_alumnado3).

Estas acciones metodológicas y organizativas contribuyen a que el alumnado comprenda la diversidad ingénita que existe en los diferentes grupos humanos y, muy sustancialmente, a percibir esa diversidad como una ocasión para el aprendizaje y el enriquecimiento.

Alumna: Es una actividad que sirve para estar todos participando y para apoyar a los demás.

Alumna: Somos una mezcla heterogénea.

Investigadora: ¿qué significa eso?

Alumna: Que hay una mezcla, que hay una diversidad. (GD_alumnado2).

\section{Acción tutorial... "en el ámbito personal, escolar, profesional y social"}

Observamos cómo el centro trata de abordar estas dimensiones haciendo converger diferentes acciones: análisis de las propuestas curriculares, generación de actividades específicas y, esencialmente, el proyecto de las tutorías compartidas. Cada curso, durante las tutorías compartidas, el alumnado encuentra un espacio de desarrollo de su identidad y de reflexión sobre las diversas esferas a través de un asunto de relevancia social: convivencia, educación emocional, interculturalidad o género.

Estos temas sirven como ejes organizadores de las propuestas y permiten abordar todas las dimensiones anteriormente declaradas. La siguiente imagen ilustra cómo durante el curso escolar 2017-2018 la escuela desarrolla acciones vinculadas con la igualdad de género. 


\section{Figura 1.}

Las dimensiones de la Acción Tutorial en el proyecto de las Tutorías Compartidas (igualdad de género).

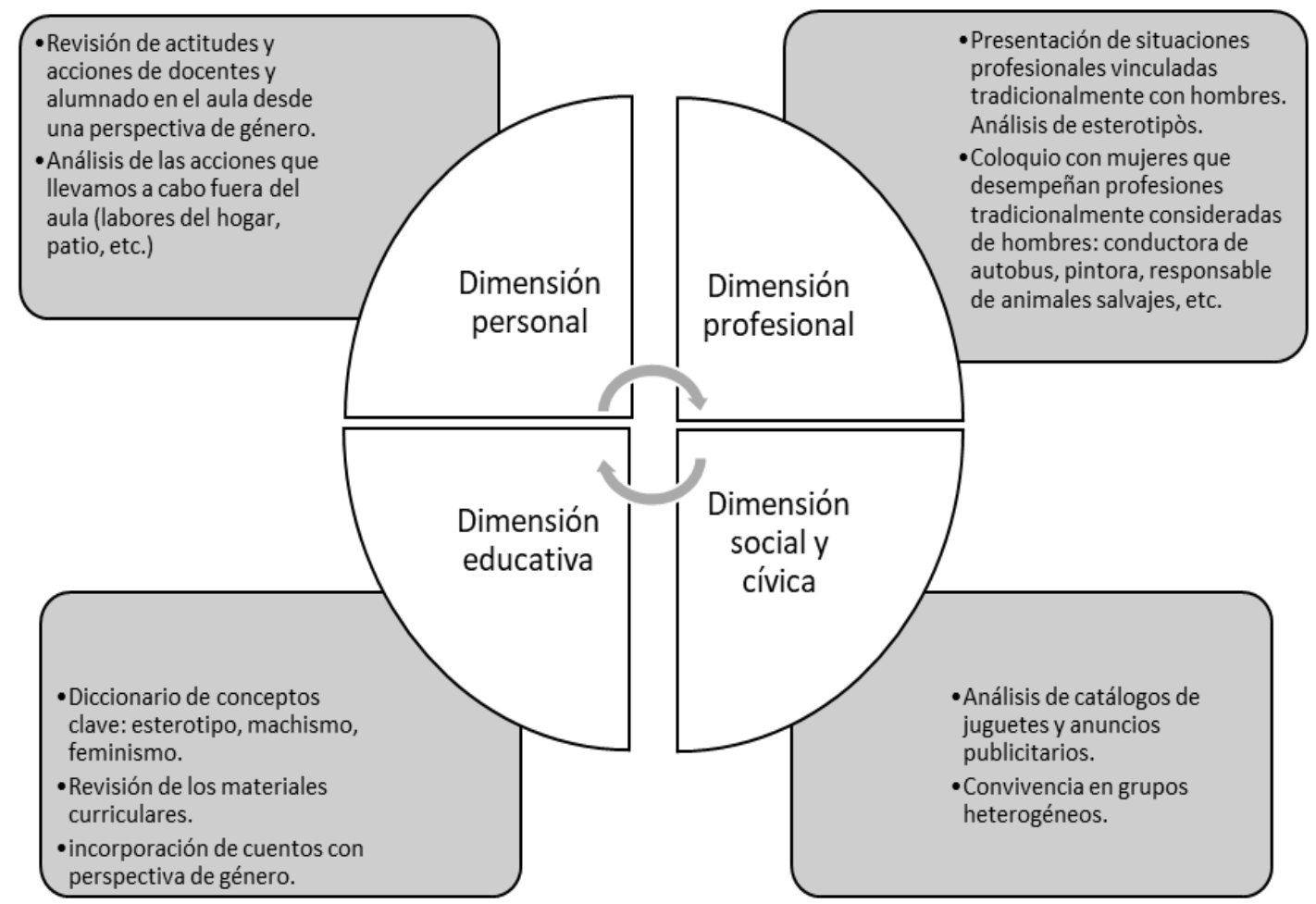

Fuente: Elaboración propia.

Dadas las limitaciones de espacio, nos detendremos en dos de ellas que han sido menos trabajadas en la literatura: la dimensión socio-cívica y la profesional.

La primera tiene como propósito acompañar a los alumnos en el proceso de construcción de su propia identidad como ciudadanos en el marco de una sociedad compleja, diversa y democrática. Todos los asuntos elegidos surgen de una sensibilidad especial del centro por lo que acontece dentro y más allá de sus paredes. Por ejemplo, cuando se eligió el tema de la interculturalidad, en los medios de comunicación se establecía la discusión sobre la inmigración y los refugiados provenientes de la guerra de Siria. Igualmente, el asunto de la igualdad de género se desarrolló en paralelo a los movimientos sociales de igualdad como el "Me too", las manifestaciones feministas en España, etc.

Tiene el interés normal en una problemática actual [...] Quizá porque en ese momento era una problemática que a nivel social estaba muy presente, los medios de comunicación estaban bombardeando y, además, estábamos trabajando los ODS y uno de los 17 objetivos aboga por trabajar la igualdad de género. (Entrevista_tutora).

La propia organización de los grupos de alumnos busca promover la convivencia de alumnado de diferentes edades, procedencias y características, tal y como hemos explicado.

"Me parece muy bien porque estamos todos juntos, los niños más mayores con los más pequeños. $Y$ aunque haya cosas más difíciles los mayores nos ayudan a hacerlo". (GD_alumnado3).

Finalmente, centramos el análisis en el ámbito profesional. La orientación vocacional y/o profesional ha sido tradicionalmente uno de los ejes centrales de la acción tutorial, especialmente 
en la Educación Secundaria Obligatoria. Para abordar esta dimensión es habitual que se diseñen actividades que ofrecen al alumnado la oportunidad de conocer sus fortalezas y competencias con el fin de orientar sus decisiones académicas y profesionales. Sin embargo, en estos procesos no se produce un análisis de los sistemas de producción actual ni de las relaciones laborales.

En las tutorías compartidas se aborda esta dimensión desde una perspectiva más compleja y con una clara mirada crítica e inclusiva. Trata de superar la mirada reduccionista de la orientación profesional incorporando al debate y análisis asuntos tales como la desigualdad laboral, la brecha de género, el reparto de las tareas, etc.

Pequeños comentarios que salen en el aula, como qué queremos ser de mayores, y ves que un niño, en este caso un niño gitano, que él quiere de mayor ser peluquero y no pasa nada. $Y$ una niña que quiere ser jugadora de rugby, porque ha visto un ejemplo que seguir. (GD_docentes2).

\section{Acción tutorial... "que ha de contemplar la colaboración del resto de agentes educativos"}

En relación con las familias, durante las tutorías compartidas se trata de trascender la colaboración puntual habitual en las escuelas (Méndez et al., 2010; Ceballos y Saiz, 2019) para considerarlas como aliadas irremplazables en el desarrollo del proyecto. Emergen diferentes acciones y espacios de vinculación y trabajo conjunto que permiten materializar este axioma del que la escuela parte.

En ocasiones, participan directamente en las sesiones, protagonizando el desarrollo de las tutorías compartidas:

Los docentes nos habían relatado en nuestras conversaciones informales que las familias conocen y colaboran con la escuela. En la sesión de hoy, vienen mujeres que realizan trabajos que rompen con los estereotipos de género. Algunas de ellas forman parte de la red local, otras de la red personal de los docentes, y varias de ellas son familiares de los niños y niñas del centro. "La conductora de camiones era una madre de un niño", me dice la maestra. (Diario de investigación).

Es imprescindible señalar que su presencia no es una apuesta cosmética, sino el resultado de un trabajo conjunto. En encuentros previos, los tutores muestran a las familias el sentido y temática de la propuesta y les invitan a participar. Desde este momento, son las familias quienes, con diferentes grados de autonomía, seleccionan el contenido de su intervención, los recursos a utilizar y el modo de trabajo con el alumnado. Esto sitúa a los tutores como apoyos de las familias.

Por otro lado, encontramos una participación indirecta a través de sus hijos/as, quienes se convierten en mediadores entre sus contextos más próximos: la escuela y la familia.

En algunos sí ha habido cambios. Me encontré con él un día en el recreo y me dijo: jo, le he dicho a mi padre que por qué no fregábamos nosotros que mamá la pobre... y me ha dicho mi padre: "pero tú estás "chinao", pero qué dices..." Ha ido a su casa y se ha encontrado con la barrera que teníamos, pero sin duda, ha habido un cambio (Entrevista tutora).

Vemos cómo los dos escenarios de desarrollo del alumnado entran en diálogo, mediados por el alumnado. Aquello que sucede en la escuela, lo aprendido durante las tutorías compartidas, es proyectado en la familia. Así, el proyecto ayuda al alumnado a interrogarse sobre su entorno más próximo y a proponer alternativas. Pero el proceso es bidireccional, de modo que las preguntas que se realizan las familias y los modos de ser y hacer en el ámbito familiar encuentran también su espacio de diálogo en la escuela. 
Finalmente, si señalábamos que los estudiantes se convertían en mediadores del contexto familiar y escolar, podemos indicar que las familias asumen el mismo papel en relación con la comunidad local.

A mí me ha llegado información de amistades que no tienen que ver con el colegio que me preguntan: oye, qué es eso de las tutorías raras que hacéis en el colegio. Algo estaremos haciendo bien para que las familias comenten esta información fuera. Yo iba a decir que trasciende un poco más... de fuera, otros centros, el CEP está interesado en el tema. Nos llaman para que vayamos a contarlo. (GD_profesorado1).

Las familias trasladan a la comunidad la acción educativa del centro, desde su implicación y conocimiento de las tutorías compartidas, construyendo una imagen del proyecto educativo en un ámbito, el local, habitualmente alejado de la misma. Sin embargo, esta mediación no concluye aquí, sino que trasciende hacia la invitación directa a participar. De estas acciones, se desprende un modelo de acción tutorial en el que las familias se convierten en agentes indispensables del proyecto.

"Nuestro colegio es, y ha sido en su historia, una escuela hecha por y para todos los componentes de la comunidad escolar, buscando permanentemente aumentar el nivel de participación de todos sus miembros: de los alumnos tanto en las aulas como en el centro, de los profesores a través del trabajo colaborativo y de las familias y el resto del entorno en su implicación en la escuela. Por ello consideramos imprescindible que el centro sea el motor de cambio que propicie dinámicas de apertura al entorno de forma que se favorezca un intercambio bidireccional entre escuela y comunidad". (PIIE).

Por otro lado, encontramos la colaboración de la comunidad local a través de espacios de intercambio y establecimiento de relaciones (Cieza, 2010; Simón, 2011). Dar respuesta a la complejidad social requiere abandonar la inercia que señala a la escuela como respuesta de todas las demandas sociales y caminar hacia la colaboración, tejiendo corresponsabilidades socioeducativas. Esto implica un doble proceso reconocible en el proyecto analizado: la apertura de la escuela a la comunidad y realidad social y la implicación de la comunidad local en las acciones educativas de la escuela (Longás et al., 2008). De un lado, las temáticas surgidas que dan estructura al trabajo irrumpen en la escuela desde el análisis de la sociedad circundante: interculturalidad, igualdad, género, convivencia, etc.

Del otro, este proyecto busca la participación directa de la comunidad local (Simón, 2011). La escuela, en este caso desde el marco de acción tutorial, coordina el trabajo conjunto de los diferentes agentes que forman parten de la comunidad cercana.

Me reciben la orientadora y $G$. en la biblioteca, están esperando ya las participantes de la actividad junto con la directora. A medida que van llegando las van presentando. Cuando se completa el grupo, la orientadora les entrega las credenciales y les recuerda en qué consiste la actividad. Hasta este momento ellas no sabían con qué compañera iban a compartir la sesión. Se respira un aire entre alegría y nervios (Observación 4).

La comunidad se hace presente en la escuela, bien por la presencia de miembros de dicha comunidad local que prestan sus experiencias y aprendizajes al proyecto educativo, bien porque estos agentes participan de los procesos de planificación y desarrollo de su intervención, siendo ellos quienes definen su acción.

Cada una lo planteó como quiso, unas desde las anécdotas de su infancia, de lo que les decían sus padres, de si les habían ayudado... hubo gente que en las tutorías individuales de cada curso diseñaron preguntas para hacerles. (GD_profesorado2). 
Cabe señalar, finalmente, que la escuela ha ofrecido a la comunidad un espacio para compartir sus preocupaciones y para reconocer el trabajo de miembros de dicha comunidad que buscan alcanzar cuotas de mayor equidad y denunciar situaciones de exclusión. En definitiva, un proyecto que tiene como propósito último, el cambio y transformación de su entorno local.

\section{Conclusiones y discusión}

En este trabajo examinamos algunas claves que permiten tender puentes entre la definición de acción tutorial y el proyecto de tutorías compartidas desplegado en el CEIP Manuel Llano. EI centro trata de abandonar las relaciones de competitividad para transitar hacia relaciones colaborativas, sostenidas en la educación inclusiva. Resaltamos algunas acciones que pueden servir para responder a este propósito, si bien no queremos reducirlas a un recetario. Más bien, esta investigación nos muestra la complejidad e interseccionalidad de las acciones educativas para trabajar las dimensiones de acción tutorial (Álvarez, 2006; Ceballos, 2017; Gallego y Riart, 2006; Torrego et al., 2014).

Una de las principales características del proyecto de las tutorías compartidas es su vinculación con el centro al completo y su compromiso férreo con la atención a la diversidad. En consecuencia, hay dos líneas de acción centrales. En primer lugar, encontramos que el centro redefine las agrupaciones y relaciones, habitualmente vinculadas con la educación graduada y la presencia de un único tutor, para configurar grupos internivelares de $1^{\circ}$ a $6^{\circ}$ de Primaria. Su propósito es generar oportunidades de encuentro, convivencia y colaboración. Por otro lado, dado que la acción tutorial es una propuesta inherente a la actividad educativa y, por ende, vinculada a todo el profesorado (Álvarez, 2006; Blanchard, 2010), cada grupo internivelar tiene asignado uno o dos tutores diferentes al del grupo aula. Esto permite multiplicar las oportunidades de que el profesorado conozca y genere vínculos con todo el alumnado, con beneficios en cuanto a cuidado, atención u orientación en el desarrollo de las tutorías compartidas, pero también en otros momentos de la jornada escolar, como los recreos y descansos entre clases o las actividades complementarias.

Encontramos una AT que despliega acciones en el plano individual (personalización de los aprendizajes, apoyo individualizado...) y también en el grupal, tanto en los grupos-aula de referencia como en los nuevos conformados. Para tal fin, hacen uso de su autonomía escolar (Saloio y Morales, 2107) para flexibilizar los tiempos escolares y estipular un momento semanal en el que todo el centro comparte en su horario una hora de tutoría. Una hora no presente en la normativa actual pero implantada por el centro. En esta misma línea, hallamos la configuración de tiempos y espacios docentes, a través de los equipos impulsores, para diseñar las propuestas educativas, entre ellas las tutorías compartidas.

Un tercer elemento característico es la implicación de las familias como socias insustituibles para el desarrollo de la iniciativa escolar (Álvarez y Bisquerra, 2012; García-Albaladejo y SánchezLiarte, 2006; Llevot y Bernad, 2015). En el proyecto de tutorías compartidas, las familias han participado protagonizando alguna de las sesiones, pero también indirectamente a través de la intermediación de sus hijos/as. Sin embargo, queda como reto futuro incorporarlas en los procesos de análisis y mejora del proyecto de tutorías compartidas.

De hecho, esta vocación de convivencia rebasa los muros escolares y se instaura en el seno de la comunidad local, pues de esta emanan los temas organizadores de las Tutorías (Bottrell y Goodwin, 2011; Cummings y Dyson, 2007). Por otro lado, el centro busca establecer espacios de 
colaboración con la comunidad a través de entidades locales, un proceso no exento de dificultades para acoplar tiempos y objetivos.

En definitiva, el valor de esta investigación reside en su papel inspirador para mejorar las prácticas de los centros educativos y concretamente la acción tutorial. Realizarlo desde una perspectiva etnográfica nos ha permitido analizar, describir y comprender el funcionamiento de la cultura escolar desplegada en este centro y colegir algunas buenas prácticas. Estas son presentadas, no con un propósito generalizador, sino como estelas de reflexión que pueden ayudar a otros centros. Asimismo, un estudio de estas características requiere una estancia prolongada en el campo por parte de las investigadoras, así como de un tiempo extenso de análisis. Esto entra en conflicto con la política actual que premia lo instantáneo. Por último, un estudio etnográfico, precisa acceder a las narraciones y voces de los participantes. En este trabajo hemos utilizado una amplia variedad de técnicas que han incorporado, esencialmente, a los estudiantes y los maestros. Por ello, nos proponemos continuar profundizando en el conocimiento de la cultura escolar del centro incorporando al análisis nuevas voces, especialmente, las de las familias y la comunidad local.

\section{Referencias bibliográficas}

Ainscow, M., Booth T. y Dyson, A (2006). Improving schools, developing inclusion. Routledge.

Álvarez, C. (2011). El interés de la etnografía escolar en la investigación educativa. Estudios pedagógicos, 37(2), 267-279. https://doi.org/10.4067/S0718-07052011000200016

Álvarez, M. (2006). La Acción Tutorial: su concepción y su práctica. MEC.

Álvarez, M. (2017). Hacia un modelo integrador de la tutoría en los diferentes niveles educativos. Educatio siglo XXI, 35(2), 21-42. https://doi.org/10.6018///298501

Álvarez, M. y Bisquerra, R. (2012). Orientación educativa. Modelos, áreas, estrategias y recursos. Wolters Kluwe.

Arnáiz, P. (2001). "Fundamentación de la tutoría". En R. Argüís (Ed.), La acción tutorial. El alumnado toma la palabra (pp. 13-24). Graó.

Beach, D., Bagley, C. y Márquez, S. (2018). The Handbook of ethnography of education. Wiley.

Besalú, C. (2010). Hacer de la Acción Tutorial el eje nuclear de la formación individualizada a lo largo de la escolaridad obligatoria y el fundamento de la acción docente, en Colectivo Yedra, Eds. (Coord.), Libro blanco de la educación intercultural (pp. 68-70). UGT.

Blanchard, M. (2010). Considerar la tutoría como un área curricular con objetivos, contenidos y criterios de evaluación propios, incluyendo, por otro lado, las funciones específicas del tutor o de la tutora, en Colectivo Yedra, Eds. (Coord.), Libro blanco de la educación intercultural (pp. 70-73). UGT.

Bottrell, D. y Goodwin, S. (2011). Schools, Communities and Social Inclusion. MacMillan.

Ceballos, N (2017). La tutoría como espacio de democracia: una mirada desde la voz de los docentes en formación. Revista Española de Orientación y Psicopedagogía, 28(1), 86-103. https://doi.org/10.5944/reop.vol.28.num.1.2017.19360 
Ceballos, N. y Saiz, Á. (2019). La acción tutorial como escenario de colaboración familiaescuela. REOP-Revista Española de Orientación y Psicopedagogía, 30(2), 28-45. https://doi.org/10.5944/reop.vol.30.num.2.2019.25336

Cieza, J. (2010). El compromiso y la participación comunitaria de los centros escolares. Un nuevo espacio-tiempo de intervención socioeducativa. Revista Interuniversitaria de Pedagogía Social, 17, 123-136. https://www.redalyc.org/articulo.oa?id=135013577010

Cotán, A. (2020). El método etnográfico como construcción de conocimiento: un análisis descriptivo sobre su uso y conceptualización en ciencias sociales. Márgenes, Revista de Educación de la Universidad de Málaga, 1(1), 83-103. https://doi.org/10.24310/mgnmar.v1i1.7241

Cummings, C. y Dyson, A. (2007). The role of school in area regeneration. Research papers in Education, 22(1), 1-22. https://doi.org/10.1080/02671520601152029

Expósito, J. (2014). La Acción Tutorial en la educación actual. Síntesis.

Flick, U. (2018). Designing Qualitative Research. SAGE Publications.

Gallego, S. y Riart, J. (2006). La tutoría y la orientación en el siglo XXI: nuevas propuestas. Octaedro Editorial.

García-Albaladejo, A. y Sánchez-Liarte, C. (2006). La participación de los padres en los documentos institucionales de los centros educativos. En C. Sánchez (Ed.), Participación de las familias en la vida escolar: acciones y estrategias (p. 149-181). Ministerio de Educación.

Galve, J. (2002). Orientación y Acción Tutorial. CEPE.

Kamberelis, G., Dimitriadis, G. y Welker, A. (2017). Focus Group Research and/in Figured Worlds. En N. Denzin y Y. Lincoln (Ed.), The SAGE handbook of qualitative research (p. 692-716). Sage Publications.

Kvale, S. (2011). Las entrevistas en investigación cualitativa. Morata.

Lara, A. (2008). La función tutorial. Un reto en la educación de hoy. Grupo Editorial Universitario.

Lázaro, A., y Asensi, J. (1989). Manual de orientación y tutoría. Narcea.

Llevot, N., y Bernad, O. (2015). La participación de las familias en la escuela: factores clave. RASE: Revista de la Asociación de Sociología de la Educación, 8(1), p. 57-70. https://ojs.uv.es/index.php/RASE/article/view/8761

Longás, J. et al. (2008). Escuela, educación y territorio. La organización en red local como estructura innovadora de atención a las necesidades socioeducativas de una comunidad.

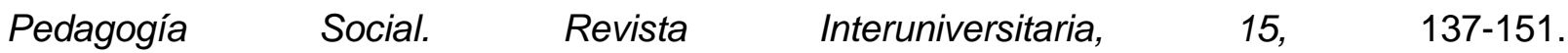
https://doi.org/10.7179/PSRI 2008.15.11

Méndez, L. et al. (2010). La tutoría en educación infantil. Cisspraxis.

Mollá, N. y Longás, J. (2007). La escuela orientadora: La Acción Tutorial desde una perspectiva institucional. Narcea.

Torrego, J. et al. (2014). 8 ideas clave. La tutoría en los centros educativos. Editorial Graó.

Monescillo, M. (2015). Orientación psicopedagógica, educación y televisión. Comunicar, 13, 25. https://www.revistacomunicar.com/ojs/index.php/comunicar/article/view/C25-2005-184 
O'Connor, J. (2019). Document analysis. En M. Lambert (Ed.), Practical Research Methods in Education: An Early Researcher's Critical Guide (p. 67-75). Routledge.

Parrilla, Á., Muñoz-Cadavid, M. y Sierra, S. (2013). Proyectos educativos con vocación comunitaria. Revista de Investigación en Educación, 11(3), 15-31.

Restrepo, E. (2018). Etnografía: alcances, técnicas y éticas. Universidad Nacional Mayor de San Marcos.

Rist, R. (1980). Blietzkrieg Ethnography: on the transformation of a method into a movement. Educational Researcher, 9, 8-10, 1980. https://doi.org/10.3102/0013189X009002008

Rozsahegyi, T. (2019). Observations. En: M. Lambert (Ed.), Practical Research Methods in Education: An Early Researcher's Critical Guide (pp. 23-34). Routledge.

Saloio, M. y Morales, J. (2017). Class councils: Organization, leadership y autonomy. Revista Lusofona de Educacao, 35(35), 101-118.

Santana, L. (2012). Orientación educativa e intervención psicoeducativa. Cambian los tiempos, cambian las responsabilidades profesionales. Pirámide.

Sanz, R. (2010). El profesor como tutor: un reto a consolidar en el ejercicio profesional de la orientación. Revista española de orientación y psicopedagogía, 21(2), 346-357. https://doi.org/10.5944/reop.vol.21.num.2.2010.11537

Serra, C. (2004). Etnografía escolar, etnografía de la educación. Revista de Educación, 334, 165176.

Simón, A. (2011). Apostando por una comunidad educadora. Aula de infantil, 61, 21-24.

Woods, P. (1987). La escuela por dentro. La etnografía en la investigación educativa. Paidós.

Fecha de entrada: 29 febrero 2020

Fecha de revisión: 25 mayo 2020

Fecha de aceptación: 28 mayo 2020 